\title{
A SIMPLE PROOF ON THE NON-EXISTENCE OF SHRINKING BREATHERS FOR THE RICCI FLOW
}

\author{
SHu-Yu Hsu \\ Department of Mathematics \\ National Chung Cheng University \\ 168 University Road, Min-Hsiung \\ Chia-Yi 621, Taiwan, R.O.C. \\ e-mail:syhsu@math.ccu.edu.tw
}

Jan 16, 2006

\begin{abstract}
Suppose $M$ is a compact n-dimensional manifold, $n \geq 2$, with a metric $g_{i j}(x, t)$ that evolves by the Ricci flow $\partial_{t} g_{i j}=-2 R_{i j}$ in $M \times(0, T)$. We will give a simple proof of a recent result of Perelman on the non-existence of shrinking breather without using the logarithmic Sobolev inequality.
\end{abstract}

It is known that Ricci flow is a very powerful tool in understanding the geometry and structure of manifolds. In 1982 R. Hamilton [H1] first began the study of Ricci flow on a manifold. Suppose $M$ is a compact 3 -dimensional manifold with a metric $g_{i j}(x)$ having a strictly positive Ricci curvature. R. Hamilton proved that if the metric $g_{i j}$ evolve by the Ricci flow

$$
\frac{\partial}{\partial t} g_{i j}=-2 R_{i j}
$$

with $g_{i j}(x, 0)=g_{i j}(x)$, then the evolving metric will converge modulo scaling to a metric of constant positive curvature. A similar result for compact 4-dimensional manifold with positive curvature operator was proved by R. Hamilton in the paper [H2]. By using a modification of the proof of Li-Yau Harnack inequality [LY] for the heat equations on manifolds R. Hamilton [H4] proved the Harnack inequality for the Ricci flow. Singularities of solutions of the Ricci flow was studied by R. Hamilton [H5] and G. Perelman [P1], [P2].

Ricci flow on non-compact manifolds was studied by W.X. Shi [S1], [S2], R. Hamilton [H3], and L.F. Wu [W1], [W2]. Existence and asymptotic behaviour of solutions of the Ricci flow equation on non-compact $\mathbb{R}^{2}$ was studied by S.Y. Hsu in the papers [Hs1-4].

1991 Mathematics Subject Classification. Primary 58J35, 53C44 Secondary 58C99.

Key words and phrases. Ricci flow, monotonicity of infinitely many functional, non-existence of shrinking breathers. 
We refer the reader to the paper [H5] by R. Hamilton and the book [CK] B. Chow and D. Knopf for various recent results on the Ricci flow. One can also read the recent lecture notes by $\mathrm{B}$. Chow $[\mathrm{C}]$ on Ricci flow.

A metric $g_{i j}(t)$ evolving by the Ricci flow in $M \times(0, T)$ is called a steady (shrinking, expanding respectively) breather if there exist $0<t_{1}<t_{2}<T$ and $\alpha=1(0<\alpha<1$, $\alpha>1$ respectively) and a diffeomorphism $\phi: M \rightarrow M$ such that $g_{i j}\left(t_{2}\right)=\phi^{*}\left(\alpha g_{i j}\left(t_{1}\right)\right)$. As observed by G. Perelman [P1] if one considers Ricci flow as a dynamical system on the space of Riemannian metrics modulo diffeomorphism and scaling, then breathers correspond to periodic orbits for the Ricci flow. So it is interesting to know whether breather exists in a Ricci flow.

In the paper [P1] G. Perelman found two functionals for the Ricci flow which are monotone increasing with respect to time. G. Perelman then used these and logarithmic Sobolev inequality to proved that there is no expanding or shrinking breathers for the Ricci flow. However his proof of non-existence of shrinking breathers has some gaps and requires the existence of solution of some auxillary parabolic equation on a manifold with initial value a delta mass which is highly non-trivial. In this paper we will modify Perelman's argument and give a simple proof of the non-existence of shrinking breathers without using the logarithmic Sobolev inequality.

The plan of the paper is as follows. In section 1 we will prove some technical lemmas. In section 2 we will fix the gaps in the proof of the monotonicity property of the $W$ functional in Perelman's paper [P1]. We will also prove the non-existence of shrinking breathers.

We will assume that $M$ is a compact n-dimensional manifold, $n \geq 2$, with a metric $g(t)=\left(g_{i j}(\cdot, t)\right)$ that evolves by the Ricci flow $(0.1)$ in $M \times(0, T)$ for the rest of the paper.

\section{Section 1}

In this section we will establish some technical lemmas. We first recall a standard result (cf. Theorem 1.6.2 of $[\mathrm{J}]$ ).

Lemma 1.1. Let $0<t_{1}<T$ and $f \in C^{\infty}\left(M \times\left(0, t_{1}\right)\right)$. For any $t \in\left(0, t_{1}\right)$ there exist a smooth function $\psi_{s}^{t}(p)=\psi^{t}(p, s): M \times\left(0, t_{1}\right) \rightarrow M$ satisfying

$$
\left\{\begin{aligned}
\frac{\partial}{\partial s} \psi^{t}(p, s) & =-\nabla f\left(\psi^{t}(p, s), t\right) & & \forall s \in\left(0, t_{1}\right), p \in M \\
\psi^{t}(p, 0) & =p & & \forall p \in M .
\end{aligned}\right.
$$

By an argument similar to the proof of Theorem 1.6.2 of $[\mathrm{J}]$ we have the following lemma.

Lemma 1.2. Let $0<t_{1}<T$ and $f \in C^{\infty}\left(M \times\left(0, t_{1}\right)\right)$. For any $t_{0} \in\left(0, t_{1}\right)$ there exist a smooth function $\phi_{t_{0}}(p, t)=\phi_{t_{0}, t}(p)$ such that $\phi_{t_{0}}: M \times\left(0, t_{1}\right) \rightarrow M$ and satisfies

$$
\left\{\begin{aligned}
\frac{\partial}{\partial t} \phi_{t_{0}}(p, t) & =-\nabla f\left(\phi_{t_{0}}(p, t), t\right) & & \forall t \in\left(0, t_{1}\right), p \in M \\
\phi_{t_{0}}\left(p, t_{0}\right) & =p & & \forall p \in M .
\end{aligned}\right.
$$

If $t_{0}^{\prime} \in\left(0, t_{1}\right)$, then the map $\phi_{t_{0}, t_{0}^{\prime}}: M \rightarrow M$ is a diffeomorphism with inverse $\phi_{t_{0}^{\prime}, t_{0}}$. 
Lemma 1.3. Let $0<t_{1}<T, t, t_{0} \in\left(0, t_{1}\right), \delta_{0}=\min \left(t, t_{1}-t\right)$, and let $f, \psi_{s}^{t}, \phi_{t_{0}, t}$ be as in Lemma 1.1 and Lemma 1.2. Let $p \in M$ and $x=\left(x_{1}, \ldots, x_{n}\right): U \subset M \rightarrow \mathbb{R}^{n}$ be a local co-ordinate chart around $p_{0}=\phi_{t_{0}, t}(p)$ for some open neighbourhood $U$ of $p_{0}$ such that $x(U)=B_{R_{0}}$ for some $R_{0}>0$ and $x\left(\phi_{t, t+h}\left(p_{0}\right)\right), x\left(\psi_{h}^{t}\left(p_{0}\right)\right) \in B_{R_{0}}$ for any $|h| \leq \delta_{1}$ for some constant $0<\delta_{1} \leq \delta_{0}$. Let $e(h)=x\left(\phi_{t, t+h}\left(p_{0}\right)\right)-x\left(\psi_{h}^{t}\left(p_{0}\right)\right)$ for any $|h| \leq \delta_{1}$. Then there exists a constant $C>0$ such that

$$
|e(h)|+\max _{1 \leq k \leq n}\left|\frac{d e_{k}}{d h}\right|+\max _{1 \leq j, k \leq n}\left\{\left|\left(\frac{\partial}{\partial x_{j}}\right)_{p_{0}} e_{k}\right|+\left|\frac{d}{d h}\left(\frac{\partial}{\partial x_{j}}\right)_{p_{0}} e_{k}\right|\right\} \leq C|h| \quad \forall|h| \leq \delta_{1}
$$

where $e(h)=\left(e_{k}(h)\right)_{k=1}^{n}$ in this local co-ordinate system and $|e(h)|=\left(\sum_{k=1}^{n} e_{k}(h)^{2}\right)^{1 / 2}$.

Proof. Without loss of generality we will abuse the notation and write $\phi_{t, t+h}\left(p_{0}\right), \psi_{h}^{t}\left(p_{0}\right)$, instead of $x\left(\phi_{t, t+h}\left(p_{0}\right)\right), x\left(\psi_{h}^{t}\left(p_{0}\right)\right)$, etc. and we will write $\partial / \partial x_{j}$ for $\left(\partial / \partial x_{j}\right)_{p_{0}}$. Let $\phi_{t, t+h}\left(p_{0}\right)=\left(\phi_{t, t+h}^{k}\left(p_{0}\right)\right)_{k=1}^{n}$ and $\psi_{h}^{t}\left(p_{0}\right)=\left(\left(\psi_{h}^{t}\right)^{k}\left(p_{0}\right)\right)_{k=1}^{n}$ in the local co-ordinate system $(x, U)$ and let

$$
q(s)=s \phi_{t, t+h}\left(p_{0}\right)+(1-s) \psi_{h}^{t}\left(p_{0}\right) \quad \forall 0 \leq s \leq 1
$$

By (1.1) and (1.2),

$$
\begin{aligned}
& \left|\frac{d e_{k}}{d h}\right| \\
= & \left|\frac{d}{d h} \phi_{t, t+h}^{k}\left(p_{0}\right)-\frac{d}{d h}\left(\psi_{h}^{t}\right)^{k}\left(p_{0}\right)\right| \\
= & \mid-g^{k j}\left(\phi_{t, t+h}\left(p_{0}\right), t+h\right) \frac{\partial}{\partial x_{j}} f\left(\phi_{t, t+h}\left(p_{0}\right), t+h\right) \\
& \quad+g^{k j}\left(\psi_{h}^{t}\left(p_{0}\right), t\right) \frac{\partial}{\partial x_{j}} f\left(\psi_{h}^{t}\left(p_{0}\right), t\right) \mid \\
\leq & \left|g^{k j}\left(\phi_{t, t+h}\left(p_{0}\right), t\right) \frac{\partial}{\partial x_{j}} f\left(\phi_{t, t+h}\left(p_{0}\right), t\right)-g^{k j}\left(\phi_{t, t+h}\left(p_{0}\right), t+h\right) \frac{\partial}{\partial x_{j}} f\left(\phi_{t, t+h}\left(p_{0}\right), t+h\right)\right| \\
& +\left|g^{k j}\left(\psi_{h}^{t}\left(p_{0}\right), t\right) \frac{\partial}{\partial x_{j}} f\left(\psi_{h}^{t}\left(p_{0}\right), t\right)-g^{k j}\left(\phi_{t, t+h}\left(p_{0}\right), t\right) \frac{\partial}{\partial x_{j}} f\left(\phi_{t, t+h}\left(p_{0}\right), t\right)\right| \\
\leq & C|h|+\left|\int_{0}^{1} \frac{d}{d s}\left(g^{k j}(q(s), t) \frac{\partial}{\partial x_{j}} f(q(s), t)\right) d s\right| \\
\leq & C|h|+\left|\int_{0}^{1} e_{i}\left(\frac{\partial}{\partial x_{i}} g^{k j}(q(s), t) \frac{\partial}{\partial x_{j}} f(q(s), t)+g^{k j}(q(s), t) \frac{\partial^{2}}{\partial x_{i} \partial x_{j}} f(q(s), t)\right) d s\right| \\
\leq & C(|h|+|e|)
\end{aligned}
$$


Hence

$$
\begin{aligned}
& \left|\frac{d|e|^{2}}{d h}\right| \leq C\left(|e|^{2}+h^{2}\right) & \forall|h| \leq \delta_{1} \\
\Rightarrow & \left|\frac{d}{d h}\left(e^{-C h}|e(h)|^{2}\right)\right| \leq C h^{2} e^{-C h} & \forall|h| \leq \delta_{1} \\
\Rightarrow & |e(h)|^{2} \leq C^{\prime} h^{2} & \forall|h| \leq \delta_{1} .
\end{aligned}
$$

Similarly

$$
\left|\frac{d}{d h}\left(\frac{\partial e_{k}}{\partial x_{j}}\right)\right| \leq C(|h|+|e|) \leq C|h| \Rightarrow\left|\frac{\partial e_{k}}{\partial x_{j}}\right| \leq C|h| \quad \forall|h| \leq \delta_{1}, j, k=1,2, \ldots, n .
$$

By (1.5), (1.6), and (1.7) we get (1.3) and the lemma follows.

Lemma 1.4. Let $0<t_{1}<T, t_{0} \in\left(0, t_{1}\right)$, and let $f$, $\phi_{t_{0}, t}$, be as in Lemma 1.2. Let

$$
\bar{g}(t)=\phi_{t_{0}, t}^{*}(g(t)) \quad \forall 0<t<t_{1} .
$$

Then

$$
\frac{\partial}{\partial t} \bar{g}(t)=\phi_{t_{0}, t}^{*}\left(\frac{\partial}{\partial t} g(t)+L_{V(t)}(g(t))\right) \quad \forall 0<t<t_{1}
$$

where $V(t)=-\nabla f(\cdot, t)$.

Proof. Let $p \in M, t \in\left(0, t_{1}\right)$, and let $\psi_{s}^{t}$ be as in Lemma 1.1. Let $(x, U), \delta_{1}>0$, and $e(h)=\left(e_{k}(h)\right)_{k=1}^{n}$ be as in Lemma 1.3. Let $X$ and $Y$ be two vector fields on $M$. Then there exist a constant $\delta_{2} \in\left(0, \delta_{1}\right)$ and an open neighbourhood $V \subset U$ of the curve $t^{\prime} \rightarrow \phi_{t_{0}, t^{\prime}}(p)$, $t-\delta_{2} \leq t^{\prime} \leq t+\delta_{2}$, such that $x(V)$ is convex in $\mathbb{R}^{n}$ and the vector fields $d \phi_{t_{0}, t^{\prime}}(X(p))$ and $d \phi_{t_{0}, t^{\prime}}(Y(p))$ along the curve $t^{\prime} \rightarrow \phi_{t_{0}, t^{\prime}}(p), t-\delta_{2} \leq t^{\prime} \leq t+\delta_{2}$, can be extended to two local vector fields $\widetilde{X}$ and $\widetilde{Y}$ on $V$. That is

$$
\begin{cases}\widetilde{X}\left(\phi_{t_{0}, t^{\prime}}(p)\right)=d \phi_{t_{0}, t^{\prime}}(X(p)) & \forall t^{\prime} \in\left(t-\delta_{2}, t+\delta_{2}\right) \\ \widetilde{Y}\left(\phi_{t_{0}, t^{\prime}}(p)\right)=d \phi_{t_{0}, t^{\prime}}(Y(p)) & \forall t^{\prime} \in\left(t-\delta_{2}, t+\delta_{2}\right) .\end{cases}
$$

Let $p_{0}=\phi_{t_{0}, t}(p)$ and

$$
\begin{array}{r}
E(h)=g\left(\phi_{t_{0}, t+h}(p), t\right)\left(d \phi_{t_{0}, t+h}(X(p)), d \phi_{t_{0}, t+h}(Y(p))\right) \\
-g\left(\psi_{h}^{t}\left(p_{0}\right), t\right)\left(d \psi_{h}^{t}\left(\widetilde{X}\left(p_{0}\right)\right), d \psi_{h}^{t}\left(\widetilde{Y}\left(p_{0}\right)\right)\right) .
\end{array}
$$

Let $\phi_{t, t+h}=\left(\phi_{t, t+h}^{k}\right)_{k=1}^{n}$ and $\psi_{h}^{t}=\left(\left(\psi_{h}^{t}\right)^{k}\right)_{k=1}^{n}$ in the local co-ordinate system $(x, U)$. We write

$$
\left\{\begin{array}{l}
\widetilde{X}(q)=a^{i}(q) \frac{\partial}{\partial x_{i}} \\
\widetilde{Y}(q)=b^{i}(q) \frac{\partial}{\partial x_{i}}
\end{array}\right.
$$


and let $q(s)=\left(q(s)^{k}\right)_{k=1}^{n}$ be given by (1.4). Since $\phi_{t_{0}, t+h}=\phi_{t, t+h} \circ \phi_{t_{0}, t}$ on $M$,

$$
\begin{aligned}
E(h)= & g\left(\phi_{t, t+h}\left(p_{0}\right), t\right)\left(d \phi_{t, t+h}\left(\widetilde{X}\left(p_{0}\right)\right), d \phi_{t, t+h}\left(\widetilde{Y}\left(p_{0}\right)\right)\right) \\
& -g\left(\psi_{h}^{t}\left(p_{0}\right), t\right)\left(d \psi_{h}^{t}\left(\widetilde{X}\left(p_{0}\right)\right), d \psi_{h}^{t}\left(\widetilde{Y}\left(p_{0}\right)\right)\right) \\
= & g_{i j}\left(\phi_{t, t+h}\left(p_{0}\right), t\right) \frac{\partial \phi_{t, t+h}^{i}}{\partial x_{k}}\left(p_{0}\right) \frac{\partial \phi_{t, t+h}^{j}}{\partial x_{k^{\prime}}}\left(p_{0}\right) a^{k}\left(p_{0}\right) b^{k^{\prime}}\left(p_{0}\right) \\
& -g_{i j}\left(\psi_{h}^{t}\left(p_{0}\right), t\right) \frac{\partial\left(\psi_{h}^{t}\right)^{i}}{\partial x_{k}}\left(p_{0}\right) \frac{\partial\left(\psi_{h}^{t}\right)^{j}}{\partial x_{k^{\prime}}}\left(p_{0}\right) a^{k}\left(p_{0}\right) b^{k^{\prime}}\left(p_{0}\right) \\
= & \int_{0}^{1} \frac{d}{d s}\left[g_{i j}(q(s), t) \frac{\partial q(s)^{i}}{\partial x_{k}} \frac{\partial q(s)^{j}}{\partial x_{k^{\prime}}} a^{k}\left(p_{0}\right) b^{k^{\prime}}\left(p_{0}\right)\right] d s \\
=e_{l}(h) & \int_{0}^{1} \frac{\partial g_{i j}}{\partial x_{l}}(q(s), t) \frac{\partial q(s)^{i}}{\partial x_{k}} \frac{\partial q(s)^{j}}{\partial x_{k^{\prime}}} a^{k}\left(p_{0}\right) b^{k^{\prime}}\left(p_{0}\right) d s \\
& +\int_{0}^{1} g_{i j}(q(s), t) \frac{\partial e_{i}}{\partial x_{k}} \frac{\partial q(s)^{j}}{\partial x_{k^{\prime}}} a^{k}\left(p_{0}\right) b^{k^{\prime}}\left(p_{0}\right) d s \\
& +\int_{0}^{1} g_{i j}(q(s), t) \frac{\partial q(s)^{i}}{\partial x_{k}} \frac{\partial e_{j}}{\partial x_{k^{\prime}}} a^{k}\left(p_{0}\right) b^{k^{\prime}}\left(p_{0}\right) d s \\
=E_{1}(h) & +E_{2}(h)+E_{3}(h)
\end{aligned}
$$

Let

$$
\begin{cases}G_{l}=\frac{\partial g_{i j}}{\partial x_{l}}(q(s), t) \frac{\partial q(s)^{i}}{\partial x_{k}} \frac{\partial q(s)^{j}}{\partial x_{k^{\prime}}} a^{k}\left(p_{0}\right) b^{k^{\prime}}\left(p_{0}\right) & \forall l=1,2, \ldots, n \\ H_{i}=g_{i j}(q(s), t) \frac{\partial q(s)^{j}}{\partial x_{k^{\prime}}} b^{k^{\prime}}\left(p_{0}\right) & \forall i=1,2, \ldots, n\end{cases}
$$

Then

$$
\begin{aligned}
\frac{d}{d h} E_{1}(h)=e_{l}(h) & \int_{0}^{1}\left(s \frac{d}{d h} \phi_{t, t+h}^{m}\left(p_{0}\right)+(1-s) \frac{d}{d h}\left(\psi_{h}^{t}\right)^{m}\left(p_{0}\right)\right) \frac{\partial G_{l}}{\partial x_{m}} d s \\
& +\left(\frac{d}{d h} e_{l}(h)\right) \int_{0}^{1} G_{l} d s \\
=-e_{l}(h) & \int_{0}^{1}\left(s \nabla_{m} f\left(\phi_{t, t+h}\left(p_{0}\right), t+h\right)+(1-s) \nabla_{m} f\left(\left(\psi_{h}^{t}\right)\left(p_{0}\right), t\right)\right) \frac{\partial G_{l}}{\partial x_{m}} d s \\
& +\left(\frac{d}{d h} e_{l}(h)\right) \int_{0}^{1} G_{l} d s
\end{aligned}
$$


and

$$
\begin{aligned}
& \frac{d}{d h} E_{2}(h) \\
= & \int_{0}^{1}\left(s \frac{d}{d h} \phi_{t, t+h}^{m}\left(p_{0}\right)+(1-s) \frac{d}{d h}\left(\psi_{h}^{t}\right)^{m}\left(p_{0}\right)\right) \frac{\partial H_{i}}{\partial x_{m}} \frac{\partial e_{i}}{\partial x_{k}} a^{k}\left(p_{0}\right) d s \\
& \quad+\int_{0}^{1} H_{i} \frac{d}{d h}\left(\frac{\partial e_{i}}{\partial x_{k}}\right) a^{k}\left(p_{0}\right) d s \\
=- & \int_{0}^{1}\left(s \nabla_{m} f\left(\phi_{t, t+h}\left(p_{0}\right), t+h\right)+(1-s) \nabla_{m} f\left(\left(\psi_{h}^{t}\right)\left(p_{0}\right), t\right)\right) \frac{\partial H_{i}}{\partial x_{m}} \frac{\partial e_{i}}{\partial x_{k}} a^{k}\left(p_{0}\right) d s \\
& \quad+\int_{0}^{1} H_{i} \frac{d}{d h}\left(\frac{\partial e_{i}}{\partial x_{k}}\right) a^{k}\left(p_{0}\right) d s .
\end{aligned}
$$

Hence by Lemma 1.3,

$$
\begin{aligned}
& \left|\frac{d}{d h} E_{1}(h)\right|+\left|\frac{d}{d h} E_{2}(h)\right| \\
& \leq C\left\{|e(h)|+\max _{1 \leq k \leq n}\left|\frac{d e_{k}}{d h}\right|+\max _{1 \leq j, k \leq n}\left[\left|\frac{\partial e_{k}}{\partial x_{j}}\right|+\left|\frac{d}{d h}\left(\frac{\partial e_{k}}{\partial x_{j}}\right)\right|\right]\right\} \leq C|h| \quad \forall|h| \leq \delta_{2} \\
\Rightarrow & \left.\frac{d}{d h} E_{1}(h)\right|_{h=0}=\left.\frac{d}{d h} E_{2}(h)\right|_{h=0}=0 .
\end{aligned}
$$

Similarly,

$$
\left.\frac{d}{d h} E_{3}(h)\right|_{h=0}=0 .
$$

By (1.10), (1.11), and (1.12),

$$
\left.\frac{d}{d h} E(h)\right|_{6=0}=0 .
$$


Then

$$
\begin{aligned}
\frac{d}{d t} \bar{g}(p, t)(X, Y) & =\left.\frac{d}{d h}\right|_{h=0} \phi_{t_{0}, t+h}^{*}(g(t+h))(p)(X, Y) \\
& =\left.\frac{d}{d h}\right|_{h=0} g\left(\phi_{t_{0}, t+h}(p), t+h\right)\left(d \phi_{t_{0}, t+h}(X(p)), d \phi_{t_{0}, t+h}(Y(p))\right) \\
& =\left.\frac{d}{d h}\right|_{h=0} g\left(\phi_{t_{0}, t+h}(p), t\right)\left(d \phi_{t_{0}, t+h}(X(p)), d \phi_{t_{0}, t+h}(Y(p))\right) \\
& =\left.\frac{d}{d h}\right|_{h=0} g\left(\psi_{h}^{t} \circ \phi_{t_{0}, t}(p), t\right)\left(d \psi_{h}^{t}\left(\tilde{X}\left(\phi_{t_{0}, t}(p)\right)\right), d \psi_{h}^{t}\left(\tilde{Y}\left(\phi_{t_{0}, t}(p)\right)\right)\right) \\
& +\phi_{t_{0}, t}^{*}\left(\frac{\partial}{\partial t} g(t)\right)(p)(X, Y) \\
& =L_{V(t)}(g(t))\left(\phi_{t_{0}, t}(p)\right)(\tilde{X}, \tilde{Y})+\phi_{t_{0}, t}^{*}\left(\frac{\partial}{\partial t} g(t)\right)(p)(X, Y) \\
& =L_{V(t)}(g(t))\left(\phi_{t_{0}, t}(p)\right)\left(d \phi_{t_{0}, t}(X), d \phi_{t_{0}, t}(Y)\right)+\phi_{t_{0}, t}^{*}\left(\frac{\partial}{\partial t} g(t)\right)(p)(X, Y) \\
& =\phi_{t_{0}, t}^{*}\left(L_{V(t)}(g(t))\right)(p)(X, Y)+\phi_{t_{0}, t}^{*}\left(\frac{\partial}{\partial t} g(t)\right)(p)(X, Y) \\
& =\phi_{t_{0}, t}^{*}\left(\frac{\partial}{\partial t} g(t)+L_{V}(t)(g(t))\right)(p)(X, Y)
\end{aligned}
$$

and (1.9) follows.

\section{Section 2}

In this section we will modify Perelman's argument [P1] and give a simple proof of a recent result of Perelman $[\mathrm{P} 1]$ on the non-existence of shrinking breather without using the logarithmic Sobolev inequality and the questionable existence of solution of some parabolic equation with initial data a delta mass.

Similar to [P1] for any $\tau>0, f \in C^{\infty}(M)$, and Riemannian metric $\widetilde{g}=\left(\widetilde{g}_{i j}\right)$ on $M$, let

$$
\begin{gathered}
\mathcal{F}(\widetilde{g}, f)=\int_{M}\left(R(\widetilde{g})+|\nabla f|^{2}\right) e^{-f} d V_{\widetilde{g}}, \\
W(\widetilde{g}, f, \tau)=(4 \pi \tau)^{-n / 2} \int_{M}\left\{\tau\left(R(\widetilde{g})+|\nabla f|^{2}\right)+f-n\right\} e^{-f} d V_{\widetilde{g}},
\end{gathered}
$$

and

$$
\mu(\widetilde{g}, \tau)=\inf _{\substack{f \in \mathcal{A}(\widetilde{g}, \tau) \\ 7}} W(\widetilde{g}, f, \tau)
$$


where $R(\widetilde{g})$ is the scalar curvature of $\widetilde{g}$ and

$$
\mathcal{A}(\widetilde{g}, \tau)=\left\{f \in C^{\infty}(M):(4 \pi \tau)^{-n / 2} \int_{M} e^{-f} d V_{\widetilde{g}}=1\right\}
$$

We will first prove that $\mu(\widetilde{g}, \tau)$ is well-defined.

Lemma 2.1. Let $\widetilde{g}$ be a Riemannian metric on $M$ and $\tau_{0}>0$. Then there exist constants $0<\delta<1, C_{1}>0$, and $C_{\tau_{0}}>0$ such that

$$
W(\widetilde{g}, f, \tau) \geq\left[(1-\delta) \lambda_{1}-\frac{\delta}{(4 \pi \tau)^{\frac{n}{2}}}\|R(\widetilde{g})\|_{L^{\infty}(M)}-4 \delta\right] \tau-C_{\tau_{0}}-\frac{C_{1}}{\tau^{n / 2}} \log (4 \pi \tau)
$$

holds for any $\tau \geq \tau_{0}, f \in \mathcal{A}(\widetilde{g}, \tau)$, where $\lambda_{1}$ is the first eigenvalue of the operator $R(\widetilde{g})-$ $4 \Delta_{\widetilde{g}}$. Hence $\mu(\widetilde{g}, \tau)>-\infty$ is well-defined for any $\tau>0$.

Proof. Without loss of generality we may assume that $n \geq 3$. Let $f \in \mathcal{A}(\widetilde{g}, \tau), \Phi=e^{-f / 2}$, and

$$
\bar{W}(\widetilde{g}, \Phi, \tau)=(4 \pi \tau)^{-n / 2} \int_{M}\left\{\tau\left(R(\widetilde{g}) \Phi^{2}+4|\nabla \Phi|^{2}\right)-\Phi^{2} \log \Phi^{2}\right\} d V_{\widetilde{g}}-n .
$$

Then

$$
W(\widetilde{g}, f, \tau)=\bar{W}(\widetilde{g}, \Phi, \tau)
$$

and

$$
(4 \pi \tau)^{-n / 2} \int_{M} \Phi^{2} d V_{\widetilde{g}}=1
$$

Let $\delta \in(0,1)$. By $(2.6)$ and $(2.7)$,

$$
\begin{aligned}
& W(\widetilde{g}, f, \tau) \\
= & \tau \frac{\int_{M}\left(R(\widetilde{g}) \Phi^{2}+4|\nabla \Phi|^{2}\right) d V_{\widetilde{g}}}{\int_{M} \Phi^{2} d V_{\widetilde{g}}}-\frac{\int_{M} \Phi^{2} \log \Phi^{2} d V_{\widetilde{g}}}{\int_{M} \Phi^{2} d V_{\widetilde{g}}}-n \\
\geq & (1-\delta) \tau \cdot \inf _{\substack{\psi \in C^{\infty}(M) \\
\psi \neq 0}}\left(\frac{\int_{M}\left(R(\widetilde{g}) \psi^{2}+4|\nabla \psi|^{2}\right) d V_{\widetilde{g}}}{\int_{M} \psi^{2} d V_{\widetilde{g}}}\right)-\frac{\delta \tau}{(4 \pi \tau)^{\frac{n}{2}}}\|R(\widetilde{g})\|_{L^{\infty}(M)}+I(\Phi)-n \\
\geq & (1-\delta) \tau \lambda_{1}-\frac{\delta \tau}{(4 \pi \tau)^{\frac{n}{2}}}\|R(\widetilde{g})\|_{L^{\infty}(M)}+I(\Phi)-n
\end{aligned}
$$

where $\lambda_{1}$ is the first eigenvalue of $R(\widetilde{g})-4 \Delta_{\widetilde{g}}$ and

$$
I(\Phi)=\frac{4 \delta \tau \int_{M}|\nabla \Phi|^{2} d V_{\widetilde{g}}-\int_{M} \Phi^{2} \log \Phi^{2} d V_{\widetilde{g}}}{\int_{\substack{M \\ \text { (n) }}} \Phi^{2} d V_{\widetilde{g}}}
$$


We will now use a modification of the technique of [R1], [R2], to control the term $I(\Phi)$. Choose $\varepsilon \in(0,2 /(n-2))$. By the Jensen's inequality, Sobolev inequality, and (2.7),

$$
\begin{aligned}
4 \delta \tau \int_{M}|\nabla \Phi|^{2} d V_{\widetilde{g}}-\int_{M} \Phi^{2} \log \Phi^{2} d V_{\widetilde{g}} & =4 \delta \tau \int_{M}|\nabla \Phi|^{2} d V_{\widetilde{g}}-\frac{1}{\varepsilon} \int_{M} \Phi^{2} \log \Phi^{2 \varepsilon} d V_{\widetilde{g}} \\
& \geq 4 \delta \tau \int_{M}|\nabla \Phi|^{2} d V_{\widetilde{g}}-\frac{2+2 \varepsilon}{\varepsilon} \log \|\Phi\|_{L^{2+2 \varepsilon}(M, \widetilde{g})} \\
& \geq 4 \delta \tau \int_{M}|\nabla \Phi|^{2} d V_{\widetilde{g}}-\frac{2+2 \varepsilon}{\varepsilon} \log \left(C\|\Phi\|_{H^{1}(M, \widetilde{g})}\right)
\end{aligned}
$$

where

$$
\|\Phi\|_{L^{q}(M, \widetilde{g})}=\left(\int_{M} \Phi^{q} d V_{\widetilde{g}}\right)^{1 / q}
$$

for any $q \geq 1$ and

$$
\|\Phi\|_{H^{1}(M, \widetilde{g})}=\|\Phi\|_{L^{2}(M, \widetilde{g})}+\|\nabla \Phi\|_{L^{2}(M, \widetilde{g})}
$$

Let

$$
\widetilde{\Phi}=\frac{\Phi}{\|\Phi\|_{L^{2}(M, \widetilde{g})}}
$$

Then

$$
\|\widetilde{\Phi}\|_{H^{1}(M, \widetilde{g})} \geq\|\widetilde{\Phi}\|_{L^{2}(M, \widetilde{g})}=1 .
$$

By (2.7), (2.9), and (2.10), $\forall \tau \geq \tau_{0}$,

$$
\begin{aligned}
I(\Phi) & \geq 4 \delta \tau\|\widetilde{\Phi}\|_{H^{1}(M, \widetilde{g})}-\frac{2+2 \varepsilon}{\varepsilon} \frac{\log \left(C\|\widetilde{\Phi}\|_{H^{1}(M, \widetilde{g})}\|\Phi\|_{L^{2}(M, \widetilde{g})}\right)}{\|\Phi\|_{L^{2}(M, \widetilde{g})}}-4 \delta \tau \\
& \geq 4 \delta \tau\|\widetilde{\Phi}\|_{H^{1}(M, \widetilde{g})}-\frac{2+2 \varepsilon}{\varepsilon(4 \pi \tau)^{n / 2}} \log \left(C\|\widetilde{\Phi}\|_{H^{1}(M, \widetilde{g})}\right)-\frac{(1+\varepsilon) n}{\varepsilon(4 \pi \tau)^{n / 2}} \log (4 \pi \tau)-4 \delta \tau \\
& \geq 4 \delta \tau_{0}\|\widetilde{\Phi}\|_{H^{1}(M, \widetilde{g})}-\frac{2+2 \varepsilon}{\varepsilon\left(4 \pi \tau_{0}\right)^{n / 2}} \log \left(C\|\widetilde{\Phi}\|_{H^{1}(M, \widetilde{g})}\right)-\frac{(1+\varepsilon) n}{\varepsilon(4 \pi \tau)^{n / 2}} \log (4 \pi \tau)-4 \delta \tau \\
& \geq C_{\tau_{0}}^{\prime}-\frac{C_{1}}{\tau^{n / 2}} \log (4 \pi \tau)-4 \delta \tau
\end{aligned}
$$

where

$$
C_{\tau_{0}}^{\prime}=\min _{y \geq 1}\left(4 \delta \tau_{0} y-\frac{2+2 \varepsilon}{\varepsilon\left(4 \pi \tau_{0}\right)^{n / 2}} \log (C y)\right)>-\infty
$$

and

$$
C_{1}=\frac{(1+\varepsilon) n}{\varepsilon(4 \pi)^{n / 2}} .
$$

By (2.8) and (2.11) we get (2.5) with $C_{\tau_{0}}=C_{\tau_{0}}^{\prime}-n$. By taking infimum over all function $f \in \mathcal{A}(\widetilde{g}, \tau)$ in $(2.5)$ we get $\mu(\widetilde{g}, \tau)>-\infty$ for any $\tau>0$ and the lemma follows. 
Corollary 2.2. Let $\widetilde{g}$ be a Riemannian metric on $M$. Suppose the first eigenvalue of $R(\widetilde{g})-4 \Delta_{\widetilde{g}}$ is positive. Then

$$
\lim _{\tau \rightarrow \infty} \mu(\widetilde{g}, \tau)=\infty
$$

Proof. This corollary is stated without proof in [P1]. We will give a short proof of it here. We fix $\tau_{0}>0$ and choose $\delta \in(0,1)$ sufficiently small such that

$$
\left[(1-\delta) \lambda_{1}-\frac{\delta}{\left(4 \pi \tau_{0}\right)^{\frac{n}{2}}}\|R(\widetilde{g})\|_{L^{\infty}(M)}-4 \delta\right]>0
$$

By Lemma 2.1 there exist constants $C_{1}>0$ and $C_{\tau_{0}}>0$ such that (2.5) holds. Taking infimum over $f \in \mathcal{A}(\widetilde{g}, \tau)$ in $(2.5)$, we get

$$
\mu(\widetilde{g}, \tau) \geq\left[(1-\delta) \lambda_{1}-\frac{\delta}{\left(4 \pi \tau_{0}\right)^{\frac{n}{2}}}\|R(\widetilde{g})\|_{L^{\infty}(M)}-4 \delta\right] \tau-C_{\tau_{0}}-\frac{C_{1}}{\tau^{n / 2}} \log (4 \pi \tau) \quad \forall \tau \geq \tau_{0}
$$

Letting $\tau \rightarrow \infty$ the corollary follows.

Lemma 2.3. Suppose $0<t_{1}<T$ and $\bar{f} \in C^{\infty}\left(M \times\left(0, t_{1}\right)\right)$. Let $\bar{g}(t)=\left(\bar{g}_{i j}(\cdot, t)\right)$ be an evolving metric on $M$ which satisfies

$$
\frac{\partial}{\partial t} \bar{g}(t)=-2\left(R_{i j}(\bar{g}(t))+\nabla_{i}^{\bar{g}(t)} \nabla_{j}^{\bar{g}(t)} \bar{f}\right) \quad \text { in } M \times\left(0, t_{1}\right)
$$

where $\nabla_{i}^{\bar{g}(t)}$ is the covariant derivative with respect to the metric $\bar{g}(t)$. Suppose

$$
\frac{\partial \bar{f}}{\partial t}=-\Delta_{\bar{g}} \bar{f}-R(\bar{g})+\frac{n}{2 \tau} \quad \text { in } M \times\left(0, t_{1}\right)
$$

where

$$
\tau=\tau(t)=t_{0}^{\prime}-t
$$

for some constant $t_{0}^{\prime}>t_{1}$. Then $\forall t \in\left(0, t_{1}\right)$,

$$
\frac{d}{d t} W(\bar{g}(t), \bar{f}(\cdot, t), \tau)=\int_{M} 2 \tau\left|R_{i j}(\bar{g}(t))+\nabla_{i}^{\bar{g}(t)} \nabla_{j}^{\bar{g}(t)} \bar{f}-\frac{1}{2 \tau} \bar{g}_{i j}\right|^{2}(4 \pi \tau)^{-n / 2} e^{-\bar{f}} d V_{\bar{g}(t)}
$$

Proof. This result is stated without proof in [P1]. For the sake of completeness we will give a simple proof of it here. Let the metric $\widetilde{g}(t)=\left(\widetilde{g}_{i j}(t)\right)$ be given by

$$
\widetilde{g}_{i j}(t)=\frac{\bar{g}_{i j}(t)}{4 \pi \tau}
$$


Then

$$
\begin{aligned}
W(\bar{g}(t), \bar{f}(\cdot, t), \tau) & =\frac{1}{4 \pi} \mathcal{F}(\widetilde{g}(t), \bar{f}(\cdot, t))+\int_{M}(\bar{f}(p, t)-n) e^{-\bar{f}(p, t)} d V_{\widetilde{g}(t)}(p) \\
\Rightarrow \quad \frac{d}{d t} W(\bar{g}(t), \bar{f}(\cdot, t), \tau)= & \frac{1}{4 \pi} \frac{d}{d t} \mathcal{F}(\widetilde{g}(t), \bar{f}(\cdot, t))+\int_{M} \bar{f}_{t}(p, t) e^{-\bar{f}(p, t)} d V_{\widetilde{g}(t)}(p) \\
& +\int_{M}(\bar{f}(p, t)-n) \frac{\partial}{\partial t}\left(e^{-\bar{f}(p, t)} d V_{\widetilde{g}(t)}(p)\right)
\end{aligned}
$$

Now by (2.12) and (2.13),

$$
\begin{aligned}
\frac{d}{d t}\left(e^{-\bar{f}} d V_{\widetilde{g}(t)}\right) & =\left(\frac{1}{2} \widetilde{g}^{i j}\left(\widetilde{g}_{i j}\right)_{t}-f_{t}\right) e^{-\bar{f}} d V_{\widetilde{g}(t)} \\
& =\left\{\frac{1}{2}(4 \pi \tau) \bar{g}^{i j}\left(\frac{\left(\bar{g}_{i j}\right)_{t}}{4 \pi \tau}+\frac{\bar{g}_{i j}}{4 \pi \tau^{2}}\right)-\bar{f}_{t}\right\} e^{-f} d V_{\widetilde{g}(t)} \\
& =\left\{\bar{g}^{i j}\left(-\left(R_{i j}(\bar{g})+\nabla_{i}^{\bar{g}} \nabla_{j}^{\bar{g}} \bar{f}\right)+\frac{\bar{g}_{i j}}{2 \tau}\right)-\bar{f}_{t}\right\} e^{-\bar{f}} d V_{\widetilde{g}(t)} \\
& =0 .
\end{aligned}
$$

Hence by (2.16) and (2.17),

$$
\frac{d}{d t} W(\bar{g}(t), \bar{f}(\cdot, t), \tau)=\frac{1}{4 \pi} \frac{d}{d t} \mathcal{F}(\widetilde{g}(t), \bar{f}(\cdot, t))+\int_{M} \bar{f}_{t}(p, t) e^{-\bar{f}(p, t)} d V_{\widetilde{g}(t)}(p)
$$

By (2.17) and section 1.1 of [P1],

$$
\begin{aligned}
& \frac{1}{4 \pi} \frac{d}{d t} \mathcal{F}(\widetilde{g}(t), \bar{f}(\cdot, t))=-\frac{1}{4 \pi} \int_{M}<\left(\widetilde{g}_{i j}\right)_{t}, R_{i j}(\widetilde{g})+\nabla_{i}^{\bar{g}} \nabla_{j}^{\bar{g}} f>_{\tilde{g}} e^{-\bar{f}} d V_{\widetilde{g}}
\end{aligned}
$$

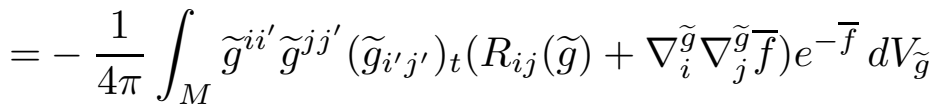

$$
\begin{aligned}
& =-(4 \pi \tau)^{-\frac{n}{2}} \int_{M} \bar{g}^{i i^{\prime}} \bar{g}^{j j^{\prime}}\left(\tau\left(\bar{g}_{i^{\prime} j^{\prime}}\right)_{t}+\bar{g}_{i^{\prime} j^{\prime}}\right)\left(R_{i j}(\bar{g})+\nabla_{i}^{\bar{g}} \nabla_{j}^{\bar{g}} \bar{f}\right) e^{-\bar{f}} d V_{\bar{g}} \\
& =(4 \pi \tau)^{-\frac{n}{2}} \int_{M}\left[2 \tau\left|\left(R_{i j}(\bar{g})+\nabla_{i}^{\bar{g}} \nabla_{j}^{\bar{g}} \bar{f}\right)\right|^{2}-\left(R(\bar{g})+\Delta_{\bar{g}} \bar{f}\right)\right] e^{-\bar{f}} d V_{\bar{g}}
\end{aligned}
$$

By (2.13), (2.18), and (2.19), we get (2.15) and the lemma follows.

Lemma 2.4. Let $H_{0} \in C^{\infty}(M)$ be such that $\min _{M} H_{0}>0$. Then for any $0<t_{1}<T$ there exists a unique solution $H \in C^{\infty}\left(M \times\left[0, t_{1}\right]\right)$ of the problem

$$
\begin{cases}H_{t}=-\Delta_{g(t)} H+R(g(t)) H & \text { in } M \times\left(0, t_{1}\right] \\ H\left(x, t_{1}\right)=H_{0}(x) & \text { in } M\end{cases}
$$


satisfying the condition

$$
H(x, t) \geq e^{-C_{2}\left(t_{1}-t\right)} \min _{M} H_{0}>0 \quad \text { in } M \times\left[0, t_{1}\right]
$$

where $C_{2}=\|R\|_{L^{\infty}\left(M \times\left[0, t_{1}\right)\right)}$.

Proof. By Theorem 6 of [H1] there exists a unique smooth solution $H \in C^{\infty}\left(M \times\left[0, t_{1}\right]\right)$ of (2.20). By continuity there exists $\delta_{1} \in\left(0, t_{1}\right)$ such that $H(x, t)>0$ on $M \times\left(t_{1}-\delta_{1}, t_{1}\right]$. Let

$$
t_{2}=\inf \left\{t^{\prime}>0: H(x, t)>0 \quad \forall x \in M, t^{\prime}<t \leq t_{1}\right\}
$$

Then $0 \leq t^{\prime} \leq t_{1}-\delta_{1}$. Suppose $t^{\prime}>0$. Let $s=t_{1}-t$. Then

$$
\begin{array}{ll}
H_{s}=\Delta H-R(g(t)) H \geq \Delta H-C_{2} H & \forall(x, s) \in M \times\left(0, t_{1}-t^{\prime}\right) \\
\Rightarrow \quad\left(e^{C_{2} s} H\right)_{s} \geq \Delta\left(e^{C_{2} s} H\right) & \forall(x, s) \in M \times\left(0, t_{1}-t^{\prime}\right)
\end{array}
$$

where $C_{2}=\|R\|_{L^{\infty}\left(M \times\left(0, t_{1}\right)\right)}$. By the maximum principle for parabolic equations,

$$
\begin{aligned}
& e^{C_{2} s} H \geq \min _{M} H_{0} & \forall(x, s) \in M \times\left[0, t_{1}-t^{\prime}\right] \\
\Rightarrow & H(x, t) \geq e^{-C_{2}\left(t_{1}-t\right)} \min _{M} H_{0}>0 & \forall(x, t) \in M \times\left[t^{\prime}, t_{1}\right] .
\end{aligned}
$$

Hence by continuity there exists a constant $\delta_{2} \in\left(0, t^{\prime}\right)$ such that $H(x, t)>0$ on $M \times\left(t^{\prime}-\right.$ $\left.\delta_{2}, t^{\prime}\right]$. This contradicts the maximality of $t^{\prime}$. Hence $t^{\prime}=0$. Putting $t^{\prime}=0$ in (2.22) we get (2.21) and the lemma follows.

Lemma 2.5. Let $f_{0} \in C^{\infty}(M)$. Then for any $0<t_{1}<T, t_{0}^{\prime}>t_{1}$, there exists a solution $f \in C^{\infty}\left(M \times\left(0, t_{1}\right)\right)$ of the problem

$$
\begin{cases}f_{t}=-\Delta_{g(t)} f+|\nabla f|^{2}-R(g(t))+\frac{n}{2 \tau(t)} & \text { in } M \times\left(0, t_{1}\right) \\ f\left(x, t_{1}\right)=f_{0}(x) & \text { in } M\end{cases}
$$

where $\tau(t)=t_{0}^{\prime}-t$.

Proof. We will use a transform of [P1] to prove the lemma. Let

$$
H_{0}(x)=\left(4 \pi\left(t_{0}^{\prime}-t_{1}\right)\right)^{-n / 2} e^{-f_{0}(x)} .
$$

Then $H_{0}>0$ on $M$. By Lemma 2.4 there exists a unique positive solution $H \in C^{\infty}(M \times$ $\left.\left(0, t_{1}\right)\right)$ of $(2.20)$. Let

$$
f(x, t)=-\log \left[(4 \pi \tau(t))^{n / 2} H\right] .
$$

Then by (2.20) $f$ satisfies (2.23). 
Theorem 2.6. For any $t_{0}^{\prime}>0, \mu\left(g(t), t_{0}^{\prime}-t\right)$ is a monotone increasing function of $t \in$ $\left(0, \min \left(t_{0}^{\prime}, T\right)\right)$. If $(M, g)$ is not a Ricci soliton, then $\mu\left(g(t), t_{0}^{\prime}-t\right)$ is a strictly monotone increasing function of $t \in\left(0, \min \left(t_{0}^{\prime}, T\right)\right)$.

Proof. Let $t_{1} \in\left(0, \min \left(t_{0}^{\prime}, T\right)\right)$. By $(2.6)$ and an argument similar to the proof in [R1], [R2], there exists a function $f_{0} \in C^{\infty}(M)$ satisfying

$$
\left(4 \pi \tau\left(t_{1}\right)\right)^{-n / 2} \int_{M} e^{-f_{0}} d V_{g\left(t_{1}\right)}=1
$$

and

$$
\mu\left(g\left(t_{1}\right), t_{0}^{\prime}-t_{1}\right)=W\left(g\left(t_{1}\right), f_{0}, t_{0}^{\prime}-t_{1}\right)
$$

where $\tau(t)$ is given by (2.14). Let $f$ be the solution of (2.23) given by Lemma 2.5. Choose $t_{0} \in\left(0, t_{1}\right)$. Let $\phi_{t_{0}, t}$ be as in Lemma $1.2, \bar{g}$ be given by $(1.8)$, and $\bar{f}(p, t)=f\left(\phi_{t_{0}, t}(p), t\right)$. Then $\bar{g}$ satisfies (1.9) with $V(t)=-\nabla f(\cdot, t)$. By $(2.24)$,

$$
\left(4 \pi \tau\left(t_{1}\right)\right)^{-n / 2} \int_{M} e^{-\bar{f}\left(p, t_{1}\right)} d V_{\bar{g}\left(t_{1}\right)}=1 .
$$

By (1.9) and (0.1),

$$
\begin{aligned}
\frac{\partial}{\partial t} \bar{g}(t) & =\phi_{t_{0}, t}^{*}\left(-2 R_{i j}(g(t))-2 \nabla_{i}^{g(t)} \nabla_{j}^{g(t)} f\right) \quad \forall 0<t<t_{1} \\
& =-2\left(R_{i j}(\bar{g}(t))+\nabla_{i}^{\bar{g}(t)} \nabla_{j}^{\bar{g}(t)} \bar{f}\right) \quad \forall 0<t<t_{1} .
\end{aligned}
$$

Hence $\bar{g}$ satisfies (2.12). By direct computation $\bar{f}$ satisfies (2.13). Hence by Lemma 2.3 (2.15) holds. Thus

$$
W\left(\bar{g}\left(t_{1}\right), \bar{f}\left(\cdot, t_{1}\right),, t_{0}^{\prime}-t_{1}\right) \geq W\left(\bar{g}(t), \bar{f}(\cdot, t),, t_{0}^{\prime}-t\right)+E\left(t, t_{1}\right) \quad \forall 0<t<t_{1}
$$

where

$$
\begin{aligned}
E\left(t, t_{1}\right) & =\int_{t}^{t_{1}} \int_{M} 2 \tau\left|R_{i j}(\bar{g}(t))+\nabla_{i}^{\bar{g}(t)} \nabla_{j}^{\bar{g}(t)} \bar{f}-\frac{1}{2 \tau} \bar{g}_{i j}\right|^{2}(4 \pi \tau)^{-n / 2} e^{-\bar{f}} d V_{\bar{g}(t)} d t \\
& =\int_{t}^{t_{1}} \int_{M} 2 \tau\left|R_{i j}(g(t))+\nabla_{i}^{g(t)} \nabla_{j}^{g(t)} f-\frac{1}{2 \tau} g_{i j}\right|^{2}(4 \pi \tau)^{-n / 2} e^{-f} d V_{g(t)} d t \\
& \geq 0
\end{aligned}
$$

with $E\left(t, t_{1}\right)>0$ if $g$ is not a Ricci soliton. Since the functional $W$ is invariant under diffeomorphism, by $(2.26) \forall 0<t<t_{1}$,

$$
\begin{aligned}
& W\left(g\left(t_{1}\right), f_{0}, t_{0}^{\prime}-t_{1}\right) \geq W\left(g(t), f(\cdot, t), t_{0}^{\prime}-t\right)+E\left(t, t_{1}\right) \\
\Rightarrow \quad & \mu\left(g\left(t_{1}\right), t_{0}^{\prime}-t_{1}\right) \geq W\left(g(t), f(\cdot, t), t_{0}^{\prime}-t\right)+E\left(t, t_{1}\right) .
\end{aligned}
$$


By direct computation,

$$
\begin{aligned}
& \frac{d}{d t}\left((4 \pi \tau(t))^{-n / 2} \int_{M} e^{-f} d V_{g(t)}\right)=0 \\
\Rightarrow \quad & (4 \pi \tau(t))^{-n / 2} \int_{M} e^{-f(p, t)} d V_{g(t)}=\left(4 \pi \tau\left(t_{1}\right)\right)^{-n / 2} \int_{M} e^{-f_{0}(p)} d V_{g\left(t_{1}\right)}=1 \quad \forall 0<t<t_{1} .
\end{aligned}
$$

Hence $f(\cdot, t) \in \mathcal{A}(g(t), \tau(t))$. Thus by $(2.27)$,

$$
\mu\left(g\left(t_{1}\right), t_{0}^{\prime}-t_{1}\right) \geq \mu\left(g(t), t_{0}^{\prime}-t\right)+E\left(t, t_{1}\right) .
$$

Since $0<t<t_{1}<\min \left(T, t_{0}^{\prime}\right)$ is arbitrary, the lemma follows.

Theorem 2.7. If $(M, g)$ is not a Ricci soliton, then there does not exist any shrinking breather for the manifold $M$ with metric $g$ evolving by the Ricci flow on $M \times(0, T)$.

Proof. Suppose $(M, g)$ is not a Ricci soliton and there exists a shrinking breather. Then there exist constants $\alpha \in(0,1), 0<t_{1}<t_{2}<T$, such that $\left(M, \alpha g\left(t_{1}\right)\right)$ is diffeomorphic to $\left(M, g\left(t_{2}\right)\right)$. Then

$$
\mu\left(\alpha g\left(t_{1}\right), \tau\right)=\mu\left(g\left(t_{2}\right), \tau\right) \quad \forall \tau>0 .
$$

Since $W(\bar{g}, f, \tau)=W(\lambda \bar{g}, f, \lambda \tau)$ for any metric $\bar{g}$ on $M$ and $\lambda>0$, for any metric $\bar{g}$ on $M$ we have

$$
\mu(\bar{g}, \tau)=\mu(\lambda \bar{g}, \lambda \tau) \quad \forall \lambda, \tau>0 .
$$

Hence by Theorem 2.6 and (2.29),

$$
\mu\left(\alpha g\left(t_{1}\right), \tau\right)=\mu\left(g\left(t_{1}\right), \tau / \alpha\right)<\mu\left(g\left(t_{2}\right),(\tau / \alpha)-\left(t_{2}-t_{1}\right)\right)
$$

Let $\tau=\alpha\left(t_{2}-t_{1}\right) /(1-\alpha)$. Then

$$
\frac{\tau}{\alpha}-\left(t_{2}-t_{1}\right)=\tau
$$

By (2.28), (2.30), and (2.31) we get a contradiction. Hence no shrinking breather exists.

\section{ReFerences}

[C] B. Chow, Lecture notes on Ricci flow I, II, III, Clay Mathematics Institute, Summer School Program 2005 on Ricci Flow, 3-Manifolds and Geometry June 20-July 16 at MSRI, http://www.claymath.org/programs/summer_school/2005/program.php\#ricci.

[CK] B. Chow and D. Knopf, The Ricci flow:An introduction, Mathematical Surveys and Monographs, Volume 110, Amer. Math. Soc., Providence, R.I., U.S.A., 2004.

[H1]R. Hamilton, Three-manifolds with positive Ricci curvature, J. Differential Geom. 17(2) (1982), 255306.

[H2]R. Hamilton, Four-manifolds with positive curvature operator, J. Differential Geom. 24(2) (1986), 153-179.

[H3]R.S. Hamilton, The Ricci flow on surfaces, Contemp. Math. 71 (1988), 237-261.

[H4]R. Hamilton, The Harnack estimate for the Ricci flow, J. Differential Geom. 37(1) (1993), $225-243$. 
[H5]R. Hamilton, The formation of singularities in the Ricci flow, Surveys in differential geometry, Vol. II (Cambridge, MA, 1993),7-136, International Press, Cambridge, MA, 1995.

[Hs1] S.Y. Hsu, Global existence and uniqueness of solutions of the Ricci flow equation, Differential and Integral Equations 14(3) (2001), 305-320.

[Hs2] S.Y. Hsu, Large time behaviour of solutions of the Ricci flow equation on $R^{2}$, Pacific J. Math. 197(1) (2001), 25-41.

[Hs3] S.Y. Hsu, Asymptotic profile of solutions of a singular diffusion equation as $t \rightarrow \infty$, Nonlinear Analysis, TMA 48 (2002), 781-790.

[Hs4] S.Y. Hsu, Dynamics of solutions of a singular diffusion equation, Advances in Differential Equations 7(1) (2002), 77-97.

[J] J. Jost, Riemannian geometry and geometric analysis, second edition, Springer-Verlag, Berlin, Heidelberg, Germany, 1998.

[LY]P. Li and S.T. Yau, On the parabolic kernel of the Schrodinger operator, Acta Math. 156 (1986), 153-201.

[P1]G. Perelman, The entropy formula for the Ricci flow and its geometric applications, http://arXiv.org/abs/math.DG/0211159.

[P2] G. Perelman, Ricci flow with surgery on three-manifolds, http://arXiv.org/abs/math.DG/0303109.

[R1]O. Rothaus, Logarithmic Solobev inequalities and the spectrum of Sturm-Liouville operators, J. Functional Analysis 39 (1980), 42-56.

[R2]O. Rothaus, Logarithmic Solobev inequalities and the spectrum of Schrödinger operators, J. Functional Analysis 42 (1981), 110-120.

[S1] W.X. Shi, Deforming the metric on complete Riemannian manifolds, J. Differential Geom. 30 (1989), $223-301$.

[S2] W.X. Shi, Ricci deformation of the metric on complete non-compact Riemannian manifolds, J. Differential Geom. 30 (1989), 303-394.

[W1]L.F. Wu, The Ricci flow on complete $R^{2}$, Comm. in Analysis and Geometry 1 (1993), 439-472.

[W2]L.F. Wu, A new result for the porous medium equation, Bull. Amer. Math. Soc. 28 (1993), 90-94.

E-MAIL ADDRESS:SYHSU@MATH.CCU.EDU.TW 